\title{
Laboreal
}

Volume $16 \mathrm{~N}^{\circ} 1 \mid 2020$

Quando o trabalho real é tabu

\section{Psicología del trabajo : una descripción general}

A psicologia do trabalho: um apanhado

La Psychologie du travail : un aperçu

The Work psychology : an Overview

\section{Jacques Leplat}

Traductor. Michelle Aslanides

\section{OpenEdition}

\section{Journals}

Edición electrónica

URL: http://journals.openedition.org/laboreal/16167

DOI: 10.4000/laboreal.16167

ISSN: 1646-5237

Editor

Universidade do Porto

\section{Referencia electrónica}

Jacques Leplat, «Psicología del trabajo : una descripción general », Laboreal [En línea], Volume 16 N01 | 2020, Publicado el 01 julio 2020, consultado el 08 julio 2020. URL : http://

journals.openedition.org/laboreal/16167 ; DOI : https://doi.org/10.4000/laboreal.16167

Este documento fue generado automáticamente el 8 julio 2020.

\section{c) (i) (8)}

Laboreal está licenciado com uma Licença Creative Commons - Atribuição-NãoComercial 4.0 Internacional. 


\title{
Psicología del trabajo : una descripción general
}

\author{
A psicologia do trabalho : um apanhado \\ La Psychologie du travail : un aperçu \\ The Work psychology : an Overview
}

Jacques Leplat

Tradución : Michelle Aslanides

\section{REFERENCIA}

Texto original [1] : Leplat, J. (1980). La psychologie du travail : un aperçu. Bulletin de Psychologie. Tome XXXIII, $n^{\circ}$ 344, 195-200.

\section{NOTA DEL EDITOR}

Traducción : Michelle Aslanides - miaslanides@gmail.com

1 Esta breve descripción general quisiera resaltar algunos rasgos importantes de la psicología del trabajo. No pretende reemplazar los textos más completos dedicados a la presentación de la disciplina que se encuentran en particular en los manuales (tomo I del Tratado de Psicología Aplicada, por ejemplo). Podrá ayudar al lector a localizar y a interrogar los textos que seguirán.

\section{Delimitación de campo}

2 La psicología del trabajo se refiere a una división de la psicología en términos del tema de estudio: entre las otras clases de esta división se encuentran, por ejemplo, la psicología escolar, la psicología deportiva, la psicología del arte. La definición del 
campo de la psicología del trabajo nos remite a la definición del trabajo. Para el psicólogo, el trabajo es esencialmente "una actividad necesaria para la realización de una tarea" (diccionario Robert). Esta actividad compleja y multifacética ha sido objeto de numerosas definiciones que resaltan varios aspectos :

- es específicamente humana (Leontiev, 1976)

- Se adquiere por aprendizaje (Ombredane y Faverge, 1955)

- Pone en juego "dos elementos interdependientes, uno de los cuales es el uso y la fabricación de herramientas (...), el segundo es que el trabajo se realiza en condiciones de actividad colectiva" (Leontiev, 1976, p. 67)

- Tiene un carácter restrictivo (Ombredane y Faverge, 1955 ; Wallon, 1946). "El único criterio común en las actividades denominadas de este modo (trabajo) es un elemento de compulsión (restricción, obligación, disciplina, etc.) que puede ser de origen interno o externo" (Friedman, 1976).

3 No nos olvidaremos de que el término de "trabajo" es polisémico. Designa a menudo a su vez el producto de la actividad, el objeto al cual se aplica la actividad y deviene así más o menos en sinónimo de obra o de tarea. Es el sentido que podemos encontrar en expresiones como "hacer su trabajo" y "sistema hombre-trabajo" o "unidad de trabajo".

De las primeras definiciones se desprende la idea que el estudio psicológico del trabajo puede hacerse con respecto a marcos de referencia variados.

El trabajador interactúa ante todo con sus instrumentos de trabajo y con su medio técnico inmediato. También forma parte de un sistema socio-técnico que engloba al primer sistema, y este sistema forma parte de sistemas más vastos, hasta el de las instituciones que definen, por ejemplo, ciertas reglas de trabajo (legislaciones diversas sobre tiempo de trabajo, sobre la seguridad, etc.) El trabajador está asimismo inserto en organizaciones (profesionales, sindicales) y en una sociedad que constituyen fuentes de determinantes específicos que explican su conducta. Tendremos aquí una base posible para un realizar un recorte de campo de estudio.

6 Se ha comenzado a hacer un repertorio de los estudios e investigaciones referidas al campo del trabajo bajo el nombre muy general de psicología aplicada o de psicotécnica. Luego el campo se diferenció y Myers propuso en 1925 el nombre de psicología industrial. Esta denominación todavía ampliamente extendida en el mundo, es sin embargo limitante ya que el trabajo no es solo industrial, pero también agrícola, comercial, administrativo, etc. El término de psicología del trabajo, más general, es entonces preferible para designar el conjunto del campo. Fue propuesto por Walther en 1926, pero tuvo poco éxito hasta un período más reciente en el que parece haber ganado terreno.

7 Podemos considerar en tanto que sectores de la psicología del trabajo a sectores definidos a partir de tipos de intervención : psicología ergonómica (a veces llamada de la ingeniería), psicología de la formación (o psico-pedagogía profesional), psicología profesional.

8 La psicología de las organizaciones que se interesa en su forma más amplia a los determinantes socio-técnicos de las conductas humanas, constituye también una parte de la psicología del trabajo. Ella manifiesta a menudo una cierta propensión a cubrir todo el campo de la psicología del trabajo, partiendo sin duda de la idea que el estudio 
de una organización en la que se ejerce un trabajo implica obligatoriamente a hacer el estudio del trabajo.

\section{Estructuración del campo}

Esta estructuración puede concebirse a partir de la psicología o a partir del trabajo : es este segundo principio el que elegimos aquí, ilustrado en la tabla I.

Tabla I - Los grandes campos de la psicología del trabajo

\begin{tabular}{|c|c|c|}
\hline Campos de estudios & $\begin{array}{c}\text { Campos de conocimientos } \\
\text { psicológicos } \\
\text { (Ejemplos) }\end{array}$ & Campos de intervención (ejemplos) \\
\hline $\begin{array}{cl}\text { Unidad individual de trabajo: } \\
\text { - } \quad \text { referida al trabajador } \\
\text { - } \quad \text { referida a las condiciones de } \\
\quad \text { trabajo } \\
\text { - } \quad \text { caracteristicas propias } \\
\text { - } & \end{array}$ & $\begin{array}{l}\text { Diferencias individuales. Aprendizaje } \\
\text { Percepción. Sensorio-motricidad. } \\
\text { Procesos cognitivos } \\
\text { Todos los conocimientos previos }\end{array}$ & $\begin{array}{l}\text { Contratación/selección. Formación. } \\
\text { Afectación Ergonomia elemental } \\
\text { Evaluación de puestos. Seguridad. }\end{array}$ \\
\hline $\begin{array}{l}\text { Unidad en su conjunto: } \\
\text { - } \quad \text { de unidades referidas al grupo } \\
\text { - } \quad \text { referidas a las condiciones de } \\
\text { - trabajo } \\
\text { - } \quad \text { características propias }\end{array}$ & $\begin{array}{l}\text { Comunicación. Trabajo en grupo } \\
\text { Estructura de la tarea. Estructura del } \\
\text { grupo } \\
\text { Todos los conocimientos previos. }\end{array}$ & $\begin{array}{l}\text { Mejora de las relaciones humanas y } \\
\text { de las comunicaciones, del clima. } \\
\text { Ergonomia de los sistemas } \\
\text { Organización del trabajo }\end{array}$ \\
\hline $\begin{array}{cl}\text { Dimensión histórica: } \\
-\quad \text { evolución de los trabajadores } \\
-\quad \text { evolución de las condiciones de } \\
\text { trabajo } \\
\text { - evolución de las unidades de } \\
\text { trabajo }\end{array}$ & $\begin{array}{l}\text { Movilidad social y profesional. } \\
\text { Envejecimiento. } \\
\text { Cambio. Problemas interculturales. } \\
\text { Todos los conocimientos previos. }\end{array}$ & $\begin{array}{l}\text { Gestión de carreras. } \\
\text { Ergonomia proyectual. } \\
\text { Planificación del empleo }\end{array}$ \\
\hline
\end{tabular}

Tabla I - Los grandes campos de la psicología del trabajo

Los campos de estudio se distinguen por su extensión y por la consideración de la dimensión histórica. Solo comentaremos aquí la primera parte distinguida. Con respecto a los campos de estudio (1era columna), a nivel de la unidad de trabajo, serán consideradas las situaciones de trabajo individuales, ya sea que se considere una de las funciones encomendadas a los trabajadores y desempeñadas por él, o se considere la totalidad de esas mismas funciones. Así es que podremos estudiar las actividades de clasificación o de mecanografía de una secretaria o la organización de todas sus actividades. La unidad de trabajo (2da columna) se puede analizar desde la perspectiva comparativa o diferencial : así es que se tienen en cuenta las características de los trabajadores en relación con su tarea (capacidad, nivel de formación, experiencia, interés, personalidad, etc.). Las metodologías utilizadas se basan en el análisis de grupos de sujetos.

11 La unidad de trabajo es también analizable en relación a las condiciones de trabajo cuyos efectos sobre la organización de la actividad serán estudiados.

Por condición de trabajo, se entenderá aquí a todas las condiciones externas a los trabajadores - instructivos, reglas de trabajo, condiciones tecnológicas, objetos y lenguajes técnicos, etc. - que se consideran en la conducta. El papel de esas condiciones 
se determinará al variarlas, en particular de acuerdo con los métodos de tipo experimental.

Finalmente, la unidad de trabajo puede ser examinada en su conjunto. Buscaremos caracterizar su estructura y funcionamiento. Podremos clasificar así la unidad en relación con varias tipologías. Por ejemplo, podremos ordenar los puestos de secretarios de acuerdo a las habilidades requeridas, a los tipos de capacitación y a los dispositivos técnicos puestos a su disposición.

Se realiza el estudio de cada uno de los tres tipos de problemas anteriores haciendo referencia a los conocimientos de la disciplina, algunas categorías de los cuales se han mencionado (2da columna) a modo de ejemplo. Estas categorías corresponden a los recortes mencionados habitualmente y bien enumerados. Los tipos de problema no son independientes, por supuesto: por ejemplo, las diferencias individuales pueden relacionarse con aspectos perceptivos. Son aún menos exhaustivos. Su única virtud aquí es la de la ilustración.

14 Finalmente, una tercera columna menciona los tipos de intervenciones que pueden utilizar los conocimientos anteriormente mencionados. Así, un estudio de las características de los conductores de camiones en un determinado contexto podrá ser utilizado para mejorar la contratación o para definir mejores programas de capacitación. Pero la elaboración de un procedimiento de contratación y de capacitación también requerirá conocimientos y habilidades extra psicológicas (de gestión, pedagógicas, tecnológicas, etc.).

15 Los campos de estudios también pueden asimismo estar constituidos por conjuntos de unidades más o menos grandes (equipo, servicio, taller, por ejemplo). Hablamos a menudo de sistemas socio-técnicos para marcar el hecho de que se trata de un conjunto de trabajadores y de condiciones técnicas (instalación, material, reglas de trabajo, etc.) en interacción. Podemos examinarlos nuevamente desde los tres aspectos mencionados anteriormente.

Finalmente podremos considerar todas las unidades anteriores desde el ángulo histórico y estudiarlas a través de su evolución.

El desglose presentado en la tabla I podría afinarse con facilidad. Las categorías que este recorte define no son independientes y de hecho representan puntos de vista sobre una misma realidad compleja. Cualquier conducta puede ser referida a cada uno de los campos definidos, pero éstos serán más o menos relevantes dependiendo de los objetivos del estudio.

\section{Psicologia del trabajo y psicologia aplicada}

La psicología del trabajo está habitualmente catalogada dentro de la psicología aplicada y para muchos es solo una psicología aplicada. Si entendemos a través de esto que está orientada esencialmente hacia la solución de problemas prácticos, tenemos una concepción errada y truncada de la psicología del trabajo. Efectivamente, lo que define entonces la aplicación, es la búsqueda de soluciones que apuntan a satisfacer un cierto número de criterios fijados (implícita o explícitamente) por las personas que solicitan el estudio. En el campo del trabajo, los criterios son de diferentes tipos: distinguimos a menudo los que conciernen muy directamente al trabajador (seguridad, satisfacción, 
salud, etc.) y los que conciernen al sistema socio-técnico (cantidad y calidad de la producción, fiabilidad, etc.)

19 Toda aplicación implica tomar en consideración algunos de esos criterios de un modo más o menos explícito. Esto se hace evidente en los estudios de validación serios. Encontraríamos ejemplos formalizados en Ombredane y Faverge (1955) y en Cronbach y Gleser (1957) respecto de la contratación y muchos otros en diferentes campos. La traducción de estos criterios en una escala monetaria permite a menudo tratarlos de manera conjunta y plantea muy claramente el problema de los pesos respectivos que debemos atribuirles. No podemos evitar ese problema en la aplicación y su solución pone inevitablemente en juego a las relaciones de fuerza sociales. Efectivamente, los actores sociales no le atribuyen los mismos pesos relativos a esos criterios y la solución de compromiso a la cual llegan es la expresión de estas relaciones. El psicólogo puede decir (jen el mejor de los casos !) que, en una situación dada, tal solución tendrá tales consecuencias (una capacitación con tal categoría de sujetos, tal método, tal duración, generará un cierto tipo de competencia) con una aproximación dada.

La elección de la solución no es de naturaleza psicológica ya que va a hacer intervenir al costo de la puesta en marcha de la capacitación, la dificultad de la contratación, el beneficio esperado del resultado para el individuo y para la organización, etc. La evaluación de la importancia relativa de estos elementos, indispensable para la elección de una solución, no es de naturaleza psicológica. El psicólogo, gracias a sus conocimientos, podrá aclarar el debate social, mostrar consecuencias de esas elecciones para los trabajadores y para el sistema, pero no está nunca calificado para definir la solución correcta.

21 Al no haber tomado conciencia claramente de estos problemas, ciertos psicólogos imputan a su disciplina las decisiones que no tienen que ver con la misma. Las reticencias por momentos manifestadas con respecto a nuestra especialidad tienen a menudo este origen. Para mostrar cuán atentos hay que estar en este punto, nos referiremos a la obra de Walther (1926). En el prefacio que redactó en esta obra, Claparède, quien es por otro lado muy buen psicólogo, se dejó llevar y escribió una cierta cantidad de reflexiones de las cuales lo menos que podemos decir es que generan una sonrisa.

“Mucho más rápido de lo que pensara quizás, el señor Walther obtiene (...) resultados maravillosos: es que la manera de trabajar de los obreros y de los capataces librados a ellos mismos es aún más irracional que lo que se supuso !! ! „... “ Lo que nos interesa es sobre todo la relación entre el rendimiento previo a la organización del trabajo y el rendimiento tras la intervención de nuestro experto psicólogo ». Luego sigue una tabla "antes-después" que Claparède comenta: "tal como lo vemos, el rendimiento se encuentra más a menudo duplicado o triplicado, por momentos incluso quintuplicado, o sextuplicado! Estas cifras (...) muestran mejor que largas frases el bello provecho que el señor Walther ha podido extraer de la organización científica del trabajo". Finalmente, el señor Walther ha sido alabado por haber sabido, durante cinco años de práctica, lograr la armonía entre patrones y obreros. Siendo capaz de ubicarse en puntos de vista opuestos en apariencia de los unos y los otros, comprendió que un trabajo digno de este nombre solo podía implicar una estrecha solidaridad de sus intereses aparentemente divergentes, y obedecer a la vez a las exigencias de naturaleza humana y a las de la producción. El principio de la racionalización del trabajo vuelto a fundarse al calor de una profunda simpatía por el trabajador, Taylor corregido por la 
psicología - tal ha sido la obra benéfica y hábil del Sr. León Walther (P. XII a XIV). Para escapar a las andanzas que manifiestan las citas anteriores y para evitar disfrazar de ciencia a prácticas que apuntan a otros objetivos, hay que esforzarse en distinguir lo que releva del ámbito de la psicología y lo que le es totalmente ajeno. Con este objetivo, el psicólogo del trabajo tiene que hacer un diagnóstico prolijo de los problemas propuestos para su estudio, evitando así adoptar sin mirada crítica la problemática de quien le solicita sus servicios.

\section{Psicología del trabajo y Psicología} objetivo es determinar las condiciones de adquisición y ejecución de los comportamientos y sus consecuencias sobre las características del sujeto de los comportamientos en los que está interesado puede dar lugar a la investigación teórica. Al igual que cualquier otro, el psicólogo estudia la forma en que un operador monitorea y corrige el funcionamiento de una instalación desde un panel de control remoto puede hacerlo con los mismos marcos teóricos y los mismos métodos que el psicólogo que estudia en el laboratorio la resolución de un rompecabezas o el juego de la torre de Hanoi

Podemos sin embargo tratar de marcar lo que hace que la psicología del trabajo sea específica dentro de la psicología. Estaremos satisfechos con un bosquejo de algunos rasgos. Las conductas a las que se interesa el psicólogo del trabajo, se relacionan con condiciones que no son construidas por él, pero que le imponen a él como a los trabajadores. Como ya hemos visto, estas conductas se relacionan con múltiples determinantes de los cuales siempre será útil evaluar al menos aproximadamente el papel relativo al comienzo de un estudio, de ahí la importancia a menudo enfatizada del análisis del trabajo. El psicólogo del trabajo siempre se ocupará de lidiar con un problema real, donde el modelo de la situación que propone explica gran parte de la variación del o de los criterios que ha elegido para caracterizar el trabajo. No deberemos olvidar tampoco que las conductas estudiadas se ejercen en condiciones que para algunas de ellas siempre serán muy difíciles, si no imposibles, de reproducir artificialmente (condiciones socioeconómicas, de organización, etc.) sean completamente originales, están sin embargo poco aborda dos en la investigación psicológica tradicional : citaremos algunos ejemplos típicos.

Los trabajadores pueden realizar su actividad entorno a la misma tarea durante períodos muy largos. Mientras que en el laboratorio un aprendizaje dura un máximo de unas pocas horas, en la empresa, el ejercicio de una misma tarea puede extenderse durante semanas, meses o incluso años. Los mecanismos de regulación de la actividad pueden verse transformados profundamente: por lo que las habilidades y los automatismos sensoriomotores y mentales cobrarán gran importancia. Su desarrollo, su funcionamiento y su posible degradación plantearán problemas importantes que a menudo escapan a la psicología de laboratorio.

Laboreal, Volume $16 \mathrm{~N}^{\circ} 1$ | 2020 
laboratorio un aspecto de la situación para estudiar los mecanismos con mayor precisión. Entonces está necesariamente obligado a examinar las relaciones de su modelo de situación con la situación misma y esto desde dos ángulos : la situación experimental es el modelo del aspecto de la situación en la que está interesado y qué fracción de las variaciones del comportamiento en la situación básica se explica por el mecanismo estudiado en la situación del modelo. Estos problemas a menudo se mencionan en la psicología del trabajo bajo el título de simulación o más ampliamente en los informes “ terreno-laboratorio » (Leplat, 1973, 1978).

\section{La evolución de la psicología del trabajo}

Terminaremos esta descripción general presentando algunas características de la evolución de la psicología del trabajo en los últimos años. Algunas de las cuales se pueden comprender concretamente mediante el examen comparativo del contenido de los libros de texto actuales y el de los de hace unos años. Un buen ejemplo está 
representado por las tres ediciones sucesivas del manual de Tiffin. El tratado de Dunette (1976) propone a otros en su introducción. El examen de las revisiones también es muy significativo. teóricos, debemos mencionar la mejora de la formación básica de los psicólogos, el desarrollo (¡todavía tímido, lamentablemente!) de la formación en psicología del trabajo en el entorno universitario, y finalmente, las comunicaciones más numerosas entre las sociedades o secciones de psicología general y del trabajo. Los congresos internacionales de la Asociación de Psicología Científica y los de la Asociación Internacional de Psicología Aplicada a menudo nos muestran que los mismos psicólogos abordan los problemas del trabajo desde el ángulo práctico en el caso de los primeros, y desde el ángulo teórico en el segundo.

41 En el capítulo que introduce su reciente tratado, Dunette (1976) confirma sus comentarios : “ los cambios en el campo de la psicología industrial y organizacional en 
el último cuarto de siglo aparecen de un modo sorprendente cuando se comparan dos manuales. El manual de 1950 (Fryer and Henry) era casi exclusivamente un manual de prácticas que enfatizaba técnicas y aplicaciones y prestaba poca atención a la investigación y a la metodología de investigación, y ninguna atención a todas las teorías del comportamiento individual $u$ organizacional. En contraste, este manual hace un marcado énfasis en las estrategias de investigación y la metodología de investigación, en teorías conductuales y pone un acento muy fuerte sobre las características y el impacto de las fuerzas e influencias socio-psicológicas que conducen a procesos de interacción entre organizaciones y personas... » (p. 2-8).

Este incremento del lugar dado a los fundamentos teóricos de la disciplina también se encuentra en la forma en que se presentan los métodos de intervención práctica. Mientras que a menudo tenían la forma de recetas en el pasado, son cada vez más el resultado "de conocimientos desarrollados a partir de investigaciones y temas" (Dunnette 1976, p. 8). Sus condiciones de aplicación se explicitan mejor de este modo y su uso es más flexible. deben ser a la vez distinguidos y articulados. Los autores que han reflexionado sobre el estatuto de la disciplina han enfatizado este carácter y esta necesidad (por ejemplo, Rodger, 1949 ; Dubin, 1976). Los objetivos teóricos y prácticos pueden tener diferentes pesos en un estudio o una intervención, dependiendo de los objetivos y condiciones de intervención. También pueden tomar diferentes pesos dependiendo del papel de los psicólogos en las instituciones donde desarrollan su actividad. Pero en todos los casos la interacción entre ambos objetivos es esencial. De hecho, la investigación difícilmente puede ignorar el alcance práctico de la teoría y los modelos que elabora, así como no puede ignorar los aspectos psicológicos de las intervenciones en el campo. Tampoco el profesional puede ignorar, sin perjuicio, teorías o al menos marcos conceptuales ofrecidos por su disciplina. Esta interacción entre los dos objetivos es una condición de progreso en ambos casos. Las teorías y los modelos de psicología ayudarán a cuestionar 
las situaciones de trabajo e, inversamente, esas mismas situaciones podrán cuestionar esas teorías y esos modelos y contribuir a delimitar su campo de relevancia.

\section{BIBLIOGRAFÍA}

Cronbach, J.L. \& Gleser, G.C. (1957). Psychological tesis and personnal decisions. University of Illinois Prese, Urbana.

Dubin, R. (1976). Theory building in applied areas (p. 1740), ln M. D. Dunette, Handbook of industrial and organizational psychology. Chicago : Rand Mac Nally College Publishing Company.

Dunette, M.D. (1976). Handbook of industrial and organizational psychology. Chicago : Rand Mac Nally College Publishing Company.

Duraffourg, J., Guerin, F., Jankowky, F., Mascot, J.C. (1977). Analyse des activités de l'homme en situation de travail. Doc du Laboratoire de Physiologie du Travail - Ergonomie du C.N.A.M.

Friedmann, G. (1976). Ou va le travai ! humain ? Le Monde (10-1-1976).

Laville, A. (1976). L'Ergonomie. Paris : PUF.

Leplat, J. (1972). La Psychologie du travail en ergonomie (pp. 61-136). ln M. Reuchlin, Traité de Psychologie Appliquée. Paris : PUF.

Leplat, J. (1973). La méthode expérimentale en psychologie appliquée. (pp. 19-62). In M. Reuchlin, Traité de Psychologie Appliquée. Tome II. Paris : PUF.

Leplat, J. (1978). L'équivalence des situations de laboratoire et de terrain. Le Travail Humain, 41, 2, 307-318.

Leplat, J. \& Cuny, X. (1977). Introduction à la Psychologie du Travail. Paris : PUF.

Leontiev, A. (1976). Le développement du psychisme. Paris : Editions Sociales.

Ombredane, A. \& Faverge, J.M. (1955). L'analyse du travail. Paris : PUF.

Reuchlin, M. (1971) Traité de Psychologie Appliquée. Tome 1, Les applications de la psychologie. Paris : PUF.

Rodger, A. (1949). What is industrial psychology. Occupational Psychology, XXIII, 3, 170-180.

Tiffin, J. \& Mc Cormick, E. J. (1967). Psychologie industrielle. Paris : PUF.

Wallon, H. (1946). Principes de psychologie appliquée. Paris : A. Colin.

Walther, L. (1946). La psychologie du travail. Geneve : Ed. du Mt Blanc.

\section{NOTAS}

1. NT: El directo de la publicación Bulletin de psychologie nos autoriza a reproducir el artículo de Jacques Leplat y a traducirlo. 